\title{
Considering even-order terms in stochastic nonlinear system modeling with respect to broadband data communication
}

\author{
O. Schmitz ${ }^{1}$, S.K. Hampel ${ }^{1}$, H. Eul ${ }^{1}$, and D. Schwingshackl ${ }^{2}$ \\ ${ }^{1}$ Leibniz Universität Hannover, Institut für Hochfrequenztechnik und Funksysteme, Appelstr. 9A, 30167 Hannover, Germany \\ ${ }^{2}$ Infineon Austria AG, Siemensstr. 2, 9500 Villach, Austria
}

\begin{abstract}
As a tradeoff between efficiency and costs modern communication systems contain a variety of components that can at least be considered weakly nonlinear. A critical element in evaluating the degree of nonlinearity of any underlying nonlinear system is the amount of undesired signal strength or signal power this system is introducing outside the transmission bandwidth. This phenomenon called spectral regrowth or spectral broadening is subject to stringent restrictions mainly imposed by the given specifications of the particular communication standard. Consequently, achieving the highest possible efficiency without exceeding the linearity requirements is one of the main tasks in system design. Starting from this challenging engineering problem there grows a certain need for specialized tools that are capable of predicting linearity and efficiency of the underlying design. Besides a multitude of methods aiming at the prediction of spectral regrowth a statistical approach in modeling and analyzing nonlinear systems offers the advantage of short processing times due to closed form mathematical expressions in terms of input and output power spectra and is therefore further examined throughout this article.
\end{abstract}

\section{Introduction}

This article focuses on the derivation and analysis of a frequency domain model capable of predicting and estimating the degree of nonlinearity for a wide class of nonlinear systems by means of its self-induced nonlinear distortion. Besides the variety of already well established approaches, like the transient analysis or the harmonic balance analysis, the model presented in this article bears the advantage of a direct mathematical relation between input and output quantities in

Correspondence to: O. Schmitz

(schmitz@hft.uni-hannover.de) terms of closed form expressions. Therefore it is basically applicable in system-level simulations.

Historically this modeling approach has grown by the need for evaluating the spectral regrowth or spectral broadening caused by power amplifiers in classical wireless RF (Radio Frequency) transmission (see Gard, 2003; Raich, 2004). Driven at highest possible output power levels especially the power amplifier reveals nonlinear transmission behaviour, which is crucial regarding vicinal communication systems in $\mathrm{RF}$ transmission. Due to the fact that initial results in this area of research have been obtained by modeling classical $\mathrm{RF}$ transmission modules, fundamental contributions mainly deal with the modeling of nonlinear systems assuming a narrowband excitation stimulus, i.e., narrowband signalling, furthermore supposing the nonlinear system to be memoryless or static (see Gard et al., 1999).

Starting from these results the intention of this article is to adapt the present narrowband modeling approach and to modify it in a way, so that it is applicable for wideband communication systems as well. This effort not only requires the pure extension of the existing mathematical approach but also the consideration of a certain system dynamics, often called system memory, that is immediately associated with the given bandwidth the system is being probed with. The article is structured as follows: In Sect. 2 we introduce the classical narrowband nonlinear system modeling approach, also known as the bandpass approach, mainly based on the work of Gard (2003) and Raich (2004). Assuming the nonlinear system to occupy static temporal behaviour, we derive the systems time-domain output signal in terms of the narrowband input stimulus. Exploiting given inherent signal statistics it is then possible to derive the systems output autocorrelation function by means of the analytical expansion of so-called statistical higher order moments (see Davenport and Root, 1997). A subsequent Fourier transform finally yields the systems output PSD (Power spectral density) in terms of the input PSD allowing for an accurate estimation

Published by Copernicus Publications on behalf of the URSI Landesausschuss in der Bundesrepublik Deutschland e.V. 


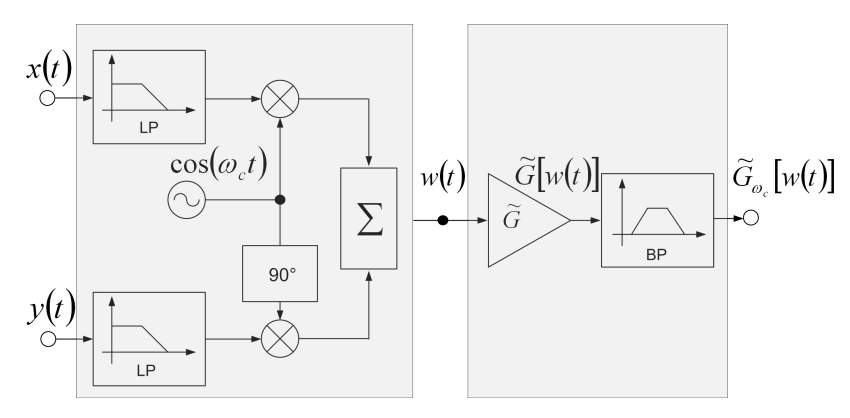

Fig. 1. Quadrature modulator and bandpass nonlinearity.

of the systems nonlinearity.

In Sect. 3 we modify the mathematical approach given in Sect. 2. Contrary to the classical narrowband assumption, we presume a broadband input signal requiring the expansion of additional higher order moments for the derivation of the autocorrelation function. As before in Sect. 2 the output PSD is obtained by applying the Fourier transform to the output autocorrelation. With regard to the input signal bandwidth we furthermore present the so-called generalized Hammerstein modeling approach, accounting for the temporal characteristics of the underlying broadband nonlinear system.

In Sect. 4 the derived frequency-domain modeling approaches are verified by time-domain simulations. For this purpose we compare mean distortion spectra computed according to the derived mathematical expressions as well as averaged distortion spectra taken from subsequent timedomain iterations for subset static and dynamic nonlinear systems.

Finally, in Sect. 5 we resume the presented results and draw essential conclusions.

\section{Narrowband modeling of nonlinear systems}

In this section we introduce the so-called bandpass modeling approach, mainly established by the work of Gard et al. (1999), Gard et al. (2001) and Zhou and Raich (2004). Fig. 1 therefore depicts a general digital transmission system followed by a static nonlinearity and a bandpass filter, whereas the combination of nonlinear system and bandpass filter is called bandpass nonlinearity for the following considerations.

At the output of the quadrature modulator the carrier signal modulated in ampltitude and phase can be stated as

$w(t)=A(t) \cos \left(\omega_{c} t+\Theta(t)\right)$

where $A(t)$ denotes the time varying amplitude, $\Theta(t)$ the time varying phase angle and $\omega_{c}$ the carrier frequency of the modulated signal in the bandpass domain. In terms of its complex envelope $\tilde{z}(t)$ we can rewrite Eq. (1)

$w(t)=\frac{1}{2} \tilde{z}(t) e^{j \omega_{c} t}+\frac{1}{2} \tilde{z}^{*}(t) e^{-j \omega_{c} t}$ with the complex envelope expressed by its in-phase component $x(t)$ and quadratue component $y(t)$ according to

$\tilde{z}(t)=A(t) e^{j \Theta(t)}=x(t)+j y(t)$.

The modulated carrier is applied to a nonlinear circuit with nonlinear gain characteristic $\tilde{G}[w(t)]$ which is assumed to be a static nonlinearity containing no significant memory within the bandwidth of modulation. Generally, a complex power series is used to model the input output relations:

$$
\begin{aligned}
\tilde{G}[w(t)] & =\tilde{a}_{1} w(t)+\tilde{a}_{2} w^{2}(t)+\cdots+\tilde{a}_{N} w^{N}(t) \\
& =\sum_{n=1}^{N} \tilde{a}_{n} w^{n}(t),
\end{aligned}
$$

where the factors $\tilde{a}_{1}$ to $\tilde{a}_{N}$ represent the generally complex coefficients taken from a $N$-th degree taylor series expansion of a nonlinear input-output characteristic for instance. Due to the subsequent bandpass zonal filtering depicted in Fig. 1 only those nonlinear terms have to be considered, that contribute to distortion components at the fundamental frequency. Since even order nonlinear terms induce distortion at baseband as well as at even multiples of the fundamental frequencies, they do not have to be considered when applying the classical narrowband bandpass analysis. Thus Eq. (4) turns into

$$
\begin{aligned}
\tilde{G}[w(t)] & =\tilde{a}_{1} w(t)+\tilde{a}_{3} w^{3}(t)+\cdots+\tilde{a}_{N} w^{N}(t) \\
& =\sum_{n=0}^{(N-1) / 2} \tilde{a}_{2 n+1} w^{2 n+1}(t),
\end{aligned}
$$

where $N$ in this case denotes the highest odd-order power contained in the polynomial description. In order to simplify the analysis a binomial expansion is applied to compute the $m$-th power of $w(t)$, yielding

$w^{m}(t)=\frac{1}{2^{m}} \sum_{k=0}^{m}\left(\begin{array}{l}m \\ k\end{array}\right) \tilde{z}(t)^{k} \tilde{z}^{*}(t)^{m-k} e^{j \omega_{c}(2 k-m) t}$.

According to the bandpass filtering depicted in Fig. 1, only the terms centered at the carrier frequency have to be taken into account. This implies $2 k-m= \pm 1$ for odd $m$ only. Substituting $k=(m+1) / 2$, the terms centered around the carrier frequency are

$w_{\omega_{c}}^{m}(t)=\left\{\frac{1}{2^{2 m-1}}\left(\begin{array}{c}m \\ \frac{m+1}{2}\end{array}\right) \tilde{z}(t)^{\frac{m+1}{2}} \tilde{z}^{*}(t)^{\frac{m-1}{2}}\right\} e^{ \pm j \omega_{c} t}$.

It is convenient to express Eq. (7) in terms of odd-order powers $m=2 n+1$ with $n=0 \ldots(N-1) / 2$ :

$w_{\omega_{c}}^{2 n+1}(t)=\left\{\frac{1}{2^{2 n}}\left(\begin{array}{c}2 n+1 \\ n+1\end{array}\right) \tilde{z}(t)^{n+1} \tilde{z}^{*}(t)^{n}\right\} e^{ \pm j \omega_{c} t}$.

The time-domain signal at the output of the bandpass nonlinearity can now be written as

$\tilde{G}_{\omega_{c}}[w(t)]=\left[\sum_{n=0}^{(N-1) / 2} \tilde{a}_{2 n+1} w_{\omega_{c}}^{2 n+1}(t)\right] e^{ \pm j \omega_{c} t}$. 
Furthermore, in terms of the input signals complex envelope $\tilde{z}(t)$ Eq. (9) can be stated as

$$
\tilde{G}_{\omega_{c}}[\tilde{z}(t)]=\sum_{n=0}^{(N-1) / 2} \frac{\tilde{a}_{2 n+1}}{2^{2 n}}\left(\begin{array}{c}
2 n+1 \\
n+1
\end{array}\right) \tilde{z}(t)^{n+1} \tilde{z}^{*}(t)^{n} .
$$

The expression in Eq. (10) describes the complex envelope of the first harmonic of a modulated carrier signal passed through a bandpass nonlinear circuit described by a complex power series. Originally this kind of description is based on a certain type of characterization of nonlinear systems. Within a so called AM-AM and AM-PM measurement campaign the amplitude of an unmodulated carrier signal is swept and the corresponding change in gain and phase at the output port of the circuit are measured at the input frequency. The amplitude gain response is known as the amplitude modulation to amplitude modulation transfer characteristic (AM-AM) and the amplitude phase response is the amplitude modulation to phase modulation characteristic (AM-PM). The fact that this way of modeling considers the determination of a complex large signal input output response clarifies the need for integrating a bandpass filter in the formal model depicted in Fig. 1.

In order to transfer the time-domain relation between input and output quantities according to Eq. (10) into a frequencydomain representation combining input and output PSDs, it is initially necessary to compute the output autocorrelation function in terms of the input autocorrelation. However, the straightforward approach we are aiming at in this article requires the assumption of certain signal statistics, that are inherently occupied by the input signal, assuming certain transmission schemes. By means of the the central limit theorem, which states that the sum of identically distributed, zero mean, independent random processes tends towards a zero mean Gaussian distribution (see Davenport and Root, 1987), it is possible to consider the input signal to be Gaussian distributed in case of multitone transmission. Hence the assumption of Gaussian statistics is quite common when focusing on multitone transmission schemes such as OFDM (Othogonal Frequency Division Multiplex) or DMT (Discrete Multitone Transmission) (see Zhou and Raich, 2004).

For further investigations the input signals complex envelope is therefore assumed to be accurately modeled by a real gaussian random process $x(t)$. The bandpass stimulus can then be expressed as

$w(t)=\frac{1}{2} x(t) e^{j \omega_{c} t}+\frac{1}{2} x(t) e^{-j \omega_{c} t}$.

The modulated carrier is applied to the input of a nonlinear system represented by the complex power series given by Eq. (5), finally yielding the time-domain output signal

$\tilde{G}_{\omega_{c}}[\tilde{z}(t)]=\sum_{n=0}^{(N-1) / 2} \frac{\tilde{a}_{2 n+1}}{2^{2 n}}\left(\begin{array}{c}2 n+1 \\ n+1\end{array}\right) x(t)^{2 n+1}$.
The output autocorrelation is then found by the expectation of the output signal

$\tilde{R}_{g g}(\tau)=E\left[\tilde{G}_{\omega_{c}}\left(\tilde{z}_{1}\right) \tilde{G}_{\omega_{c}}^{*}\left(\tilde{z}_{2}\right)\right]$,

where $\tilde{z}_{1}=\tilde{z}\left(t_{1}\right)$ and $\tilde{z}_{2}=\tilde{z}\left(t_{2}\right)=\tilde{z}\left(t_{1}-\tau\right)$. After combining Eq. (12) and Eq. (13) we obtain the expression given by Eq. (14) for the output autocorrelation.The expectation in Eq. (14) can only be evaluated if the moments of the random variable $x(t)$ are known.

$$
\begin{aligned}
\tilde{R}_{g g}(\tau)= & \sum_{n=0}^{\frac{N-1}{2}} \sum_{m=0}^{\frac{N-1}{2}} \frac{\tilde{a}_{2 n+1} \tilde{a}_{2 m+1}^{*}}{2^{2(n+m)}}\left(\begin{array}{c}
2 n+1 \\
n+1
\end{array}\right) \ldots \\
& \ldots \times\left(\begin{array}{c}
2 m+1 \\
m+1
\end{array}\right) E\left[x_{1}^{2 n+1} x_{2}^{2 m+1}\right] .
\end{aligned}
$$

In case of a zero mean real Gaussian process the moments are given by

$$
E\left[x_{1} x_{2} \ldots x_{s}\right]=\left\{\begin{array}{cl}
0 & \text { s odd } \\
\sum \prod E\left[x_{i} x_{j}\right] & \text { s even }
\end{array}\right.
$$

whereas the summation given in Eq. (15) has to be performed over all distinct pairs of subscripts $i$ and $j$ of $x$.

For many cases it is sufficient to consider the nonlinear terms of an underlying nonlinear system up to the order of $N=7$, allowing for an accurate approximation of the nonlinear transmission behaviour. However, before the relevant moments evaluated for a seventh-order power series expansion are presented, we start by denoting the statistical assumptions and having a closer look on the applied nomenclature. Assume the random variables $x_{1}$ and $x_{2}$ (whereas $x_{2}$ is a time shifted instance of $x_{1}$ ) to be Gaussian distributed with zero mean, which implies

$$
\begin{aligned}
& E\left[x_{1}\right]=E\left[x_{2}\right]=0 \\
& E\left[x_{1}^{2}\right]=E\left[x_{2}^{2}\right]=\sigma_{x_{1}}^{2}=\sigma_{x_{2}}^{2}=R_{x x}(0)=R_{x 0} .
\end{aligned}
$$

The expectation of the product of these two random variables is defined by the autocorrelation function

$E\left[x_{1} x_{2}\right]=R_{x x}(\tau)$.

Using these abbreviations Table 1 lists the moments evaluated for a seventh-order power series expansion, where only the odd-order powers corresponding to the presented bandpass approach are considered. If the Fourier transform is finally applied to the terms listed in Table 1, we obtain the output PSD in terms of its input PSD, remembering that the nfold potentiation of the autocorrelation function in the timedomain turns out to be the $n$-fold convolution of the PSD in the frequency-domain.

A verification of the derived relations in terms of simulation results is given in Sect. 4. 
Table 1. Odd order moments evaluated for a seventh order power series expansion.

\begin{tabular}{ll}
\hline Subscr. Eq. (14) & Expanded moment \\
\hline$n=0 ; m=0$ & $R_{x x}(\tau)$ \\
$n=0 ; m=1$ & $3 R_{x 0} R_{x x}(\tau)$ \\
$n=1 ; m=1$ & $9 R_{x 0}^{3} R_{x x}(\tau)+6 R_{x x}^{3}(\tau)$ \\
$n=0 ; m=2$ & $15 R_{x 0}^{2} R_{x x}(\tau)$ \\
$n=1 ; m=2$ & $45 R_{x 0}^{3} R_{x x}(\tau)+60 R_{x 0} R_{x x}^{3}(\tau)$ \\
$n=2 ; m=2$ & $225 R_{x 0}^{4} R_{x x}(\tau)+600 R_{x 0}^{2} R_{x x}^{3}(\tau)+$ \\
& $120 R_{x x}^{5}(\tau)$ \\
$n=0 ; m=3$ & $105 R_{x 0}^{3} R_{x x}(\tau)$ \\
$n=1 ; m=3$ & $315 R_{x 0}^{4} R_{x x}(\tau)+630 R_{x 0} R_{x x}^{3}(\tau)$ \\
$n=2 ; m=3$ & $1575 R_{x 0}^{5} R_{x x}(\tau)+6300 R_{x 0}^{3} R_{x x}^{3}(\tau)+$ \\
& $2520 R_{x 0} R_{x x}^{5}(\tau)$ \\
$n=3 ; m=3$ & $11025 R_{x 0}^{6} R_{x x}(\tau)+66150 R_{x 0}^{4} R_{x x}^{3}(\tau)+$ \\
& $52920 R_{x 0}^{2} R_{x x}^{5}(\tau)+5040 R_{x x}^{7 x}(\tau)$ \\
\hline
\end{tabular}

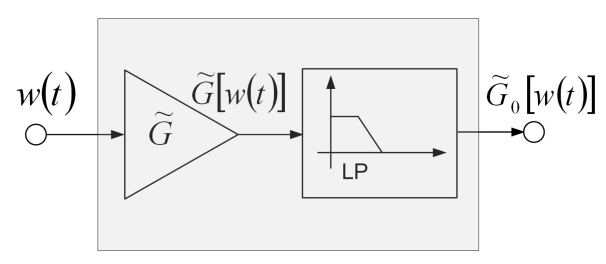

Fig. 2. Static Nonlinear system followed by an ideal low-pass filter.

\section{The broadband extension}

In case of a broadband input stimulus it is no longer sufficient to consider only the odd-order terms of a given nonlinear system, as indicated by Eq. (5). For considering evenorder terms of a power series nonlinearity as well as oddorder terms, again, we have to pick up the term denoted by Eq. (6). In contrast to the previous section we now only focus on contributions that mix into the baseband. This assumption corresponds to the idea that an ideal lowpass filter is placed right behind the nonlinear system as indicated by Fig. 2 .

That means instead of setting $2 k-m= \pm 1$ we set $2 k-m=0$ in Eq. (6), which yields

$w_{0}^{m}(t)=\frac{1}{2^{m}}\left(\begin{array}{c}m \\ \frac{m}{2}\end{array}\right) \tilde{z}(t)^{\frac{m}{2}} \tilde{z}^{*}(t)^{\frac{m}{2}}$.

Due to the assumption that $m$ can only represent even values, we can state $m=2 n$ where $n=1,2,3, \ldots, \frac{N}{2}$, provided $N$ representing the highest even-order power within the power series polynomial. Thus Eq. (18) turns into

$w_{0}^{m}(t)=\frac{1}{2^{2 n}}\left(\begin{array}{c}2 n \\ n\end{array}\right) \tilde{z}(t)^{n} \tilde{z}^{*}(t)^{n}$.

After passing through the nonlinear circuit the even-order contributions in terms of the complex envelope can be stated
Table 2. Even order moments evaluated for a sixth order power series expansion.

\begin{tabular}{ll}
\hline Subscr. Eq. (21) & Expanded moment \\
\hline$n=1 ; m=1$ & $2 R_{x x}^{2}(\tau)+R_{x 0}^{2}$ \\
$n=1 ; m=2$ & $12 R_{x x}^{2}(\tau) R_{x 0}+30 R_{x 0}^{3}$ \\
$n=1 ; m=3$ & $90 R_{x 0}^{2} R_{x x}^{2}(\tau)+15 R_{x 0}^{4}$ \\
$n=2 ; m=2$ & $24 R_{x x}^{4}(\tau)+72 R_{x x}^{2}(\tau) R_{x 0}^{2}+9 R_{x 0}^{4}$ \\
$n=2 ; m=3$ & $360 R_{x 0} R_{x x}^{4}(\tau)+540 R_{x 0}^{3} R_{x x}^{2}(\tau)+$ \\
& $45 R_{x 0}^{5}$ \\
$n=3 ; m=3$ & $720 R_{x x}^{6}(\tau)+5400 R_{x 0}^{2} R_{x x}^{4}(\tau)+$ \\
& $4050 R_{x x}^{2}(\tau) R_{x 0}^{4}+225 R_{x 0}^{6}$ \\
\hline
\end{tabular}

as

$\tilde{G}_{0}[\tilde{z}(t)]=\sum_{n=1}^{\frac{N}{2}} \frac{\tilde{a}_{2 n}}{2^{2 n}}\left(\begin{array}{c}2 n \\ n\end{array}\right) \tilde{z}(t)^{2 n} \tilde{z}^{*}(t)^{2 n}$.

Again assuming a real gaussian random process $x(t)$ modulating the carrier amplitude, the output autocorrelation function can be written as

$\tilde{R}_{g g}(\tau)=\sum_{n=1}^{\frac{N}{2}} \sum_{m=1}^{\frac{N}{2}} \frac{\tilde{a}_{2 n} \tilde{a}_{2 m}^{*}}{2^{n+m}}\left(\begin{array}{c}2 n \\ n\end{array}\right)\left(\begin{array}{c}2 m \\ m\end{array}\right) E\left[x_{1}^{2 n} x_{2}^{2 m}\right]$.

As can easily be seen from Eq. (21), corresponding to the expectation value a lot of additional statistical moments have to be evaluated when accounting for even-order distortion. As previously done in Sect. 2 the additionally expanded moments are listed in Table 2. However, in contrast to the odd order considerations Table 2 indicates that the even order terms cause a remarkable amount of distortion power at DC (Direct Current) represented by the time-domain constants given by arbitrary powers of $R_{x 0}$. In most practical applications these terms are not of considerable concern, as most transmission systems reveal an inherent highpass characteristic, therefore automatically rejecting these kind of distortion terms.

Again, as explained in the section before, the application of the Fourier transform to the specified moments given by Table 2 in combination with Eq. (21) yields the output PSD in terms of its input PSD. The employment of both mathematical approaches, therefore accounting for even and oddorder nonlinear terms, is finally capable of estimating the self-induced distortion of any given input signal, presuming the nonlinear system to occupy static temporal behaviour insofar as to be approximated according to a polynomial description.

However, this assumption can not be made in case of a broadband stimulus. In general the use of frequency selective circuitry by means of inductors or capacitors within the nonlinear device and furthermore thermal effects neccesitate a dynamic consideration of the underlying nonlinear system. 
Since the so called generalized Hammerstein model is capable of approximating a wide class of nonlinear systems with an adequate degree of accuracy, while the complexity in terms of its description parameters is relatively low, this approach is well suited for an extension of the derived model regarding the temporal characteristics (see Mathews and Sicuranza, 2000).

Figure 3 depicts the typical structure of a generalized Hammerstein system in comparison to the static polynomial model. For the extension from the static polynomial model to the generalized Hammerstein model the power series polynomial has to be split up into parallel branches, each branch representing a homogeneous nonlinearity. The dynamic behaviour is introduced by linear FIR (Finite Impulse Response) filters that follow the particular homogeneous polynomials. Finally, all branches are merged at a summing element.

Concerning the structure depicted in Fig. 3 the mathematical expressions derived previously do not change essentially. Since every branch of the parallel structure represents a static homogeneous nonlinear system, the frequency- domain model for even and odd-order nonlinear polynomials can be applied directly to the dynamic model. The subsequent filtering introduced by the linear FIR filters in every branch is then implemented in the frequency-domain by the multiplication of the filter input power spectrum (output PSD of homogenous nonlinearity) with the squared magnitude of the filter transfer function. Consequently, if $S_{n, \text { in }}(\omega)$ denotes the output PSD of a $n$th order homogenous nonlinear system in one branch of the generalized Hammerstein system thus at the same time representing the filter input PSD, the output PSD $S_{n, \text { out }}(\omega)$ of the subsequent linear FIR filter defined by its transfer function $H_{n}\left(e^{j \omega}\right)$ can be obtained by

$$
S_{n, \text { out }}(\omega)=\left|H_{n}\left(e^{j \omega}\right)\right|^{2} S_{n, \text { in }}(\omega)
$$

Thus the extension to the dynamic model does not require additional considerable effort regarding the computation of the output power specral density. Together with the derived approaches for static nonlinear systems this modeling approach is verfied in the following section by means of time-domain simulations.

\section{Simulation results}

The following section verifies the presented approaches by comparing output PSDs computed according to the derived mathematical expressions for given input PSDs with those taken from time domain-simulations for certain representative nonlinear systems. Within a time-domain simulation the given spectral mask in terms of a certain multicarrier allocation is adopted, while a random phase angle is assigned to every single tone. The time-domain signal is then obtained by performing an IFFT (Inverse Fast Fourier Transform) on

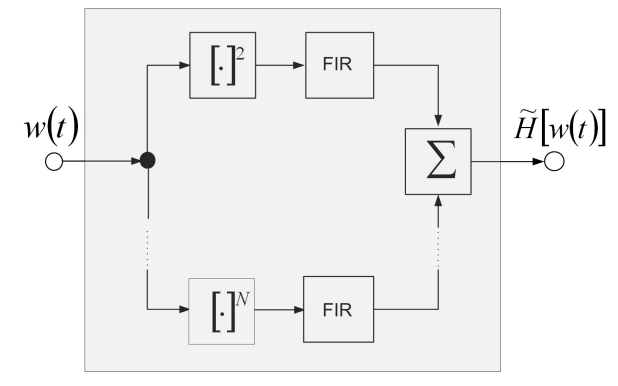

Fig. 3. Generalized Hammerstein system.

the given spectrum. The nonlinear system is finally passed by exponentiation of the time-domain signal according to the given polynomial description. The output PSD is obtainded by peforming the FFT (Fast Fourier Transform) operation. In case of dynamic systems the linear filtering is achieved by convolution with the filter impulse response.

This procedure is repeated for different choices of carrier phase sets in order to approximate a Gaussian "noiselike" stimulus. Subsequent averaging of the obtained distortion PSDs therefore yields an average output distortion PSD, comparable with those computed by the derived stochastical expressions.

First we start with a static polynomial model according to the classical bandpass approach explained in Sect. 2, hence totally described by its odd power series coefficients $\tilde{a}_{n}$. As in many practical applications, nonlinear effects shall be considered up to a degree of $N=7$. Therefore the polynomial description is given by

$\tilde{G}[w(t)]=10 w^{3}(t)+1000 w^{5}(t)+100000 w^{7}(t)$

whereas the odd coefficients are all chosen as to induce a comparable amount of distortion power. The simulation results obtained for this system are depicted in Fig. 4. Herein the frequency-domain representation of the input signal as well as the distortion PSDs obtained by time and frequencydomain simulations are given in a normalized representation. In case of multitone transmission, single tones are separated by each other corresponding to a certain carrierspacing. The normalization of the frequency axis by means of this carrierspacing yields the representation of the abscissa in terms of the depicted carrier index. The input signal (black graph) is given by two discrete "flat" frequency bands inducing the distortion PSDs computed by time-domain simulations as already described previously (blue graph) and the derived frequency-domain formula presented in Sect. 2 (red graph).

As can easily be seen, the distortion PSD obtained by means of the presented stochastical frequency-domain approach shows a good matching to the time-domain solution. The time-domain solution exhibits certain "ripples" that can be ascribed to the averaging process necessary for the approximation of a noise-like stimulus. In order to verify the mathematical modifications carried out in Sect. 3, we specify 


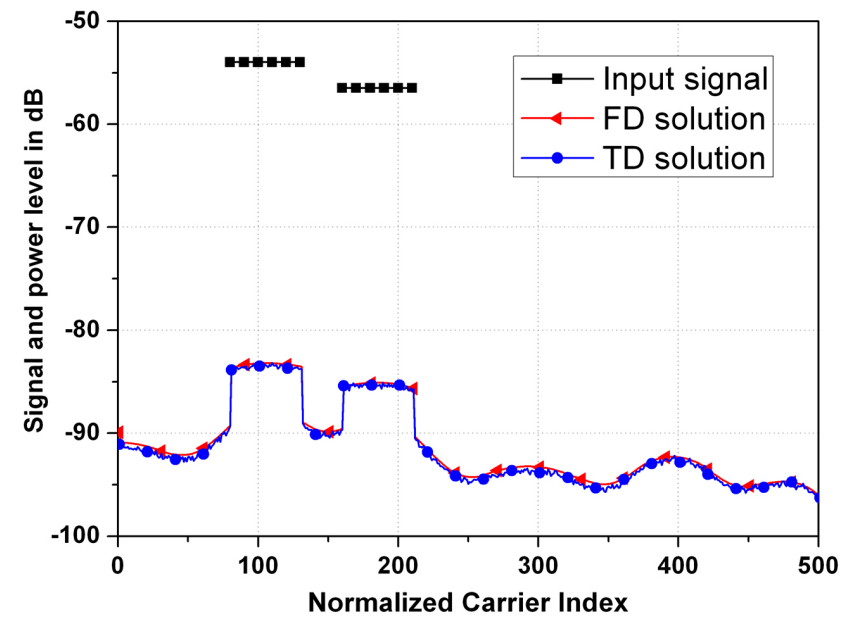

Fig. 4. Input signal and distortion PSDs computed in time and frequency domain for static odd-order model.

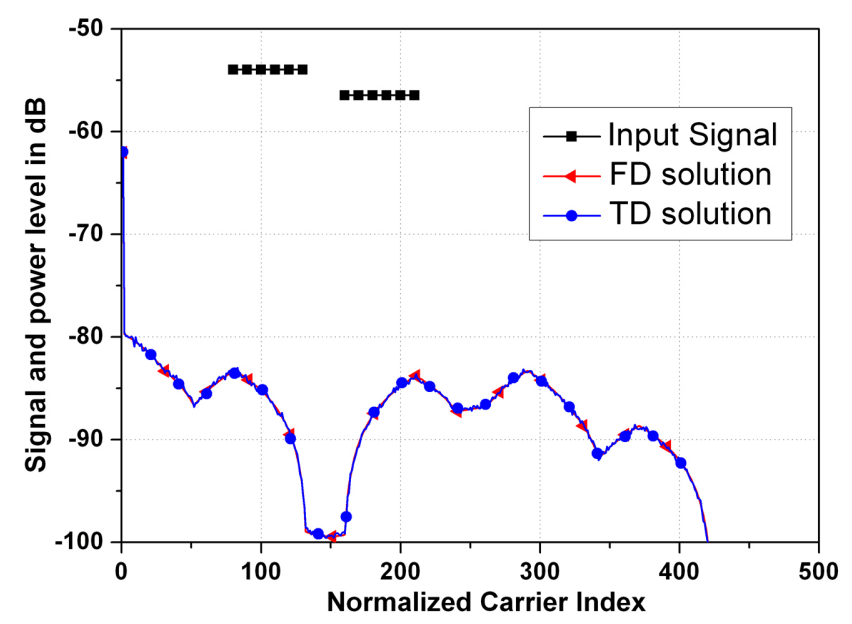

Fig. 5. Input signal and distortion PSDs computed in time and frequency domain for static even-order model.

a nonlinear polynomial model solely containing even-order terms. The chosen polynomial description is therefore given by

$$
\tilde{G}[w(t)]=w^{2}(t)+100 w^{4}(t)+10000 w^{6}(t)
$$

Figure 5 depicts the results obtained for the given even-order polynomial.

Again, we find a fairly accurate matching to the time domain iteration data. Whereas in case of the odd-order polynomial the induced in-band distortion is dominant, we recognize a remarkable amount of distortion power at the second harmonic of both input frequency bands as well as at DC for the even-order polynomial.

Simulation results in case of the dynamic Hammerstein approach are depicted in Fig. 6.

The filter coefficients needed for calculating the filter response in the frequency-domain were chosen incidentally,

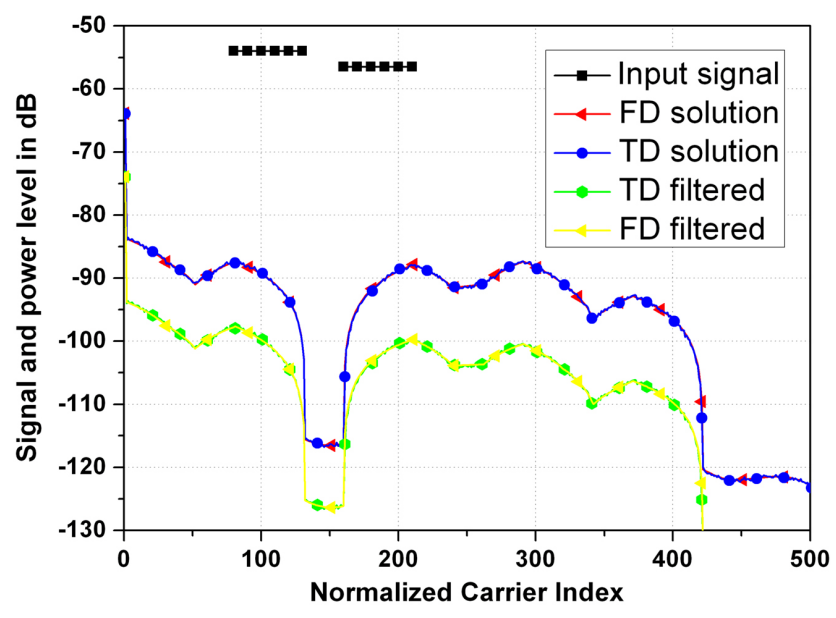

Fig. 6. Input signal and distortion PSDs computed in time and frequency domain for dynamic model corresponding to a seventh order generalized Hammerstein system.

while assuming a tap-length of $L_{t a p}=5$ for every filter branch. The underlying nonlinear system was assumed to be of maximum order $N=7$. In addition to the results obtained without the filtering operation, thus reducing the model to a pure static nonlinearity, Fig. 6 depicts the filtered output PSDs computed in time and frequency-domain. Again, even in the dynamic case, time and frequency-domain solutions show a good matching. This is not surprising at all, since the dynamic model is just adding a frequency dependent scaling corresponding to the filter response for every single nonlinear order. Beyond the accuracy achievable with the presented approaches, the derived stochastical models bear the advantage of enormously reduced processing times compared to the time domain iteration procedure. In case of the dynamic model the computation time according to the stochastic frequency-domain approach reduces by a factor of 185 opposed to time-domain simulations, as the necessity for averaging the obtained spectra vanishes.

\section{Conclusions}

In this article we introduced a stochastical frequency-domain model for the time-efficient analysis and estimation of average distortion spectra induced by nonlinear systems. In a first step we therefore presented the classical narrowband RF modeling approach and derived the fundamental formulas, necessary for the closed form analysis of output distortion PSDs in terms of given input PSDs. Furthermore, we presented a mathematical modification enabling the already established narrowband approach to cope with broadband input stimuli, accounting for even-order terms as well as for odd-order terms. Considerations on the temporal characteristics of nonlinear systems excited by broadband stimuli finally led to the introduction of the generalized Hammerstein 
system. The verification of the derived mathematical expressions was given for three representative nonlinear systems, static as well as dynamic, in terms of a comparison to iterated time-domain solutions. Herein all systems showed an accurate matching between time and frequency-domain solutions. In case of the dynamic system the required computation time could be reduced considerably. Thus the presented stochastic frequency-domain approach is a well suited candidate for the time-efficient distortion analysis of a wide class of nonlinear systems, therefore applicable in system top level simulations.

Acknowledgements. The authors are grateful with R. Stolle, Infineon Germany, for his support on the research.

\section{References}

Davenport, W. B. and Root, W. L.: An Introduction to the Theory of Random Signals and Noise, John Wiley \& Sons, Inc., 1987.
Gard, K. G.: Autocorrelation Analysis of Spectral Regrowth Generated by Nonlinear Circuits in Wireless Communication Systems, Dissertation, University of California, 2003.

Gard, K. G., Gutierrez, H. M., and Steer, M. B.: Characterization of Spectral Regrowth in Microwave Amplifiers Based on the Nonlinear Transformation of a Complex Gaussian Process, IEEE Transactions on Microwave Theory and Techniques, 47, 1999.

Gard, K. G., Steer, M. B., Larson, L. E.: Generalized Autocorrelation Analysis of Spectral Regrowth from Bandpass Nonlinear Circuits, IEEE MTT-S Digest, 2001.

Mathews, V. J. and Sicuranza, G. L.: Polynomial Signal Processing, John Wiley \& Sons, Inc., 2000.

Raich, R.: Nonlinear system Identification and Analysis with Applications to Power Amplifier Modeling and Power Amplifier Predistortion, Dissertation, Georgia Institute of Technology, 2004.

Zhou, G. T. and Raich, R.: Spectral Analysis of Polynomial Nonlinearity with Applications to RF Power Amplifiers, EURASIP Journal on Applied Signal Processing, 2004. 